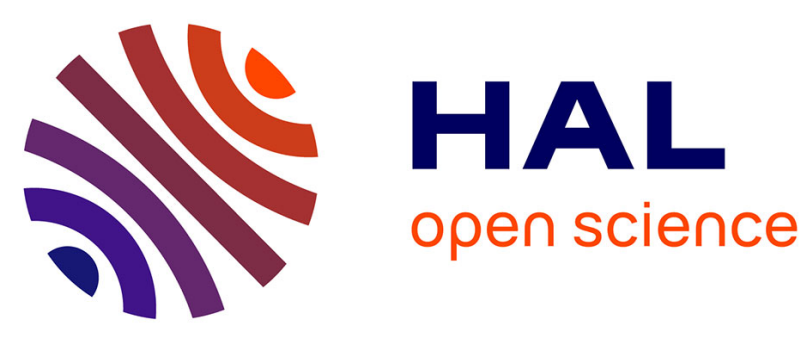

\title{
MADSTRESS: A linear approach for evaluating scattering and absorption coefficients of samples measured using time-resolved spectroscopy in reflection
}

F. Chauchard, J.M. Roger, Véronique Bellon Maurel, C. Abrahamsson, S. Andersson Engels, S. Svanberg

\section{To cite this version:}

F. Chauchard, J.M. Roger, Véronique Bellon Maurel, C. Abrahamsson, S. Andersson Engels, et al.. MADSTRESS: A linear approach for evaluating scattering and absorption coefficients of samples measured using time-resolved spectroscopy in reflection. Applied Spectroscopy, 2005, 59 (10), p. 1229 - p. 1235. 10.1366/000370205774430828 . hal-00493894

\section{HAL Id: hal-00493894 https://hal.science/hal-00493894}

Submitted on 21 Jun 2010

HAL is a multi-disciplinary open access archive for the deposit and dissemination of scientific research documents, whether they are published or not. The documents may come from teaching and research institutions in France or abroad, or from public or private research centers.
L'archive ouverte pluridisciplinaire HAL, est destinée au dépôt et à la diffusion de documents scientifiques de niveau recherche, publiés ou non, émanant des établissements d'enseignement et de recherche français ou étrangers, des laboratoires publics ou privés. 


\title{
MADSTRESS : a linear approach for evaluating scattering and absorption
} coefficients of samples measured using

\section{Time-Resolved Spectroscopy in reflection}

\author{
F. Chauchard ${ }^{\mathrm{a}, 1}$, J.M. Roger ${ }^{\mathrm{a}}, \mathrm{V}$. Bellon-Maurel ${ }^{\mathrm{a}}$, C. Abrahamsson ${ }^{\mathrm{b}}$, \\ S. Andersson-Engels ${ }^{\mathrm{b}}$ and S. Svanberg ${ }^{\mathrm{b}}$ \\ ${ }^{\mathrm{a}}$ Information and Technologies for Agro-processes - Cemagref BP 5095, 34033 \\ Montpellier Cedex 1, France \\ ${ }^{\mathrm{b}}$ Department of Physics, Lund Institute of Technology, P.O. Box 118, SE-221 00 \\ Sweden
}

\begin{abstract}
Time-resolved spectroscopy is a powerful technique permitting the separation of the scattering properties from the chemical absorption properties of a sample. The reduced scattering coefficient and the absorption coefficient are usually obtained by fitting diffusion or Monte Carlo models to the measured data using numerical optimization techniques. However, these methods do not take the spectral dimension of the data into account during the evaluation procedure, but evaluate each wavelength separately. A procedure involving multivariate methods may seem more appealing for people used to handle conventional near-infrared data. In this study we present a new method for processing TRS spectra in order to compute the absorption and reduced scattering coefficients. This approach, MADSTRESS, is based on linear regression and a $2-\mathrm{D}$ interpolation procedure. The method has allowed us to
\end{abstract}


calculate absorption and scattering coefficients of apples and fructose powder. The accuracy of the method was good enough to provide the identification of fructose absorption peaks in apple absorption spectra and the construction of a calibration model predicting the sugar content of apples.

Index Headings : Time-Resolved Spectroscopy, Equation of diffusion, Multi-linear regression, Light continuum, Apple absorption coefficient, Fructose absorption coefficient

\section{Introduction}

Near infrared (NIR) reflectance spectroscopy has the advantage that it can be used to, non-destructively, measure chemical compounds residing inside a scattering medium ${ }^{1}$. These media may be agricultural products ${ }^{2}$ or pharmaceuticals products ${ }^{3}$ or other. An example of application is the prediction of sugar content in apples ${ }^{4}$. Prominent scattering does however present a drawback as it modifies the measured absorption spectra. The measured reflectance spectrum is hence a combination of both absorption and scattering effects. Consequently, calibration models based on NIR reflectance spectra implicitly compensate for scattering effects, resulting in complex and non-robust models ${ }^{5}$. For this reason the calibration procedure is often combined with data pre-processing techniques such as Standard Normal Variate ${ }^{6}$ or Multiple Scatter Correction ${ }^{7}$. The benefits of the present pre-processing techniques are however limited since they only produce data more correlated to the absorption coefficient, but they are not capable to extract the real absorption coefficient. Furthermore the scattering coefficient can not be evaluated from

\footnotetext{
$\overline{1}$ fabien.chauchard@montpellier.cemagref.fr
} 
NIR reflectance data solely, even so its evaluation would be of deep interest, since it carries information about the physical characteristics of the sample. An attractive method for evaluating the scattering properties of highly scattering samples is time-resolved spectroscopy ${ }^{8}$ (TRS). It was mainly developed for medical applications ${ }^{9}, 10$ but has found its way into other fields of research, such as pharmaceutical ${ }^{11,12}$ and agricultural applications ${ }^{13}, 14,15$ . The parameters used to describe the optical properties of a turbid medium are the absorption coefficient $\mu_{a}(\lambda)$, the scattering coefficient $\mu_{s}(\lambda)$ and the scattering anisotropy $g$. Often, $\mu_{s}(\lambda)$ and $g$ are combined to form the reduced scattering coefficient $\mu_{s}^{\prime}(\lambda)=\mu_{s}(\lambda)(1-g)$. TRS uses short light pulses to irradiate the sample. The light diffusively re emitted by the sample at a given distance from the irradiation point is then detected as a function of time ${ }^{17}$ . TRS measurements can be conducted in either reflectance or transmission mode. In order to obtain a simultaneous measure of the temporal signal at different wavelengths, light pulses with a broad wavelength profile is used in combination with a streak camera detection system. The broad light pulses can be generated using different techniques, one is using continuum generation by focusing of a high power laser pulse into a photonic crystal fiber ${ }^{18}$.

Once the 2-dimension signal (one temporal and one spectral dimension) is recorded, the reduced scattering coefficient $\left(\mu_{s}^{\prime}(\lambda)\right)$ and the absorption coefficient $\left(\mu_{a}(\lambda)\right)$ are obtained by linking the experimental data with theoretical or modeled data. This step is crucial to get correct results, and many evaluation schemes have been proposed. Three approaches are usually used: Monte Carlo simulations $^{17,19}$, numerical optimizations ${ }^{20}, 21$ and analytical descriptors of temporal dispersion ${ }^{22}$. Those methods do however not take the spectral dimension of the data into account. $\mu_{a}(\lambda)$ and $\mu_{s}^{\prime}(\lambda)$ are calculated at a given wavelength without considering neighboring wavelengths. A method based on 
a linear solution of the equation of diffusion would allow the implementation of various chemometric tools, such as multi-linear regression (MLR), partial least square and $\mathrm{N}$-ways methods.

This study aims at proposing a new method for finding scattering and absorption coeffiecients using TRS data. The first part is devoted to the diffusion equation and a presentation of the suggested linear approach. In the second part methods and TRS instrumentation are presented. The last part describes the performance of the linear approach and its results.

\section{Theory}

When the photons of a short light pulse penetrate a turbid medium, they scatter around due to the matrix effect (Fig.1). If the light is detected at a specific distance from the light source, $\rho$, the detected light pulse will be temporally broader than the pulse sent into the medium. The dispersion of the pulse is governed by the scattering coefficient $\mu_{s}^{\prime}(\lambda)$ and the absorption coefficient $\mu_{a}(\lambda)$ of the medium. The photon transport in turbid media is described by the radiative transport equation ${ }^{23}$ which makes a balance between gained photons and lost photon. In the case of a semi-infinite homogeneous medium measured in reflectance mode, the solution is given by the time-resolved diffusion equation ${ }^{17}$ :

$$
R(t, \lambda)=(4 \pi D(\lambda) v)^{-3 / 2} z_{0}(\lambda) t^{-5 / 2} \exp \left(-\mu_{a}(\lambda) v t\right) \exp \left(-\frac{\rho^{2}+z_{0}(\lambda)^{2}}{4 D(\lambda) v t}\right)
$$

Here $R(t, \lambda)$ is the signal measured at a given distance $\rho$ at time $t, D(\lambda)$ is the diffusion coefficient with $D(\lambda)=\left[3\left(\mu_{a}(\lambda)+\mu_{s}^{\prime}(\lambda)\right)\right]^{-1}, z_{0}(\lambda)=\left(\mu_{s}^{\prime}(\lambda)\right)^{-1}$ is the mean free path of the photons and $v$ is the speed of light in the medium, 
assumed to be constant within the measured wavelength range.

\subsection{Solving the equation}

Let $t_{0}(\lambda)$ be the time where $R(t, \lambda)$ has its maximum value. By dividing the signal $R(t, \lambda)$ by its maximum value at $t_{0}(\lambda), R\left(t_{0}(\lambda), \lambda\right)$, the following equation is obtained :

$$
\begin{aligned}
\frac{R(t, \lambda)}{R\left(t_{0}(\lambda), \lambda\right)}= & \left(\frac{t}{t_{0}(\lambda)}\right)^{-5 / 2} \exp \left(\mu_{a}(\lambda) v\left(t_{0}(\lambda)-t\right)\right) \\
& \times \exp \left(-\frac{\rho^{2}+z_{0}^{2}(\lambda)}{4 D(\lambda) v t}+\frac{\rho^{2}+z_{0}^{2}(\lambda)}{4 D(\lambda) v t_{0}(\lambda)}\right)
\end{aligned}
$$

That can also be written as :

$$
\begin{aligned}
\frac{R(t, \lambda)}{R\left(t_{0}(\lambda), \lambda\right)}\left(\frac{t}{t_{0}(\lambda)}\right)^{5 / 2}= & \exp \left(\mu_{a}(\lambda) v\left(t_{0}(\lambda)-t\right)\right) \\
& \times \exp \left[\left(\frac{\left(\rho^{2}+z_{0}^{2}(\lambda)\right.}{4 D(\lambda) v}\right)\left(\frac{1}{t_{0}(\lambda)}-\frac{1}{t}\right)\right]
\end{aligned}
$$

Taking the logarithm of this equation :

$\log \left(\frac{R(t, \lambda)}{R\left(t_{0}(\lambda), \lambda\right)}\right)+\frac{5}{2} \log \left(\frac{t}{t_{0}(\lambda)}\right)=\mu_{a}(\lambda) v\left(t_{0}(\lambda)-t\right)+\left(\frac{\rho^{2}+z_{0}^{2}(\lambda)}{4 D(\lambda) v}\right)\left(\frac{1}{t_{0}(\lambda)}-\frac{1}{t}\right)$

When $R(t, \lambda)$ has its maximum, $\frac{\partial R}{\partial t}\left(t_{0}(\lambda), \lambda\right)=0 . \frac{\partial R(t, \lambda)}{\partial t}$ is given by :

$$
\begin{aligned}
\frac{\partial R(t, \lambda)}{\partial t}= & (4 \pi D(\lambda) v)^{-3 / 2} z_{0}(\lambda) \exp \left(-\mu_{a}(\lambda) v t\right) \exp \left(-\frac{\rho^{2}+z_{0}(\lambda)^{2}}{4 D(\lambda) v t}\right) \frac{5}{2} t^{-7 / 2} \\
& +(4 \pi D(\lambda) v)^{-3 / 2} z_{0}(\lambda) \exp \left(-\mu_{a}(\lambda) v t\right) \exp \left(-\frac{\rho^{2}+z_{0}(\lambda)^{2}}{4 D(\lambda) v t}\right) t^{-5 / 2} \\
& \times\left(-\mu_{a}(\lambda) v+\frac{\rho^{2}+z_{0}(\lambda)^{2}}{4 D(\lambda) v t^{2}}\right)
\end{aligned}
$$

Factorisation using $R(t, \lambda)$, the expression at $t=t_{0}(\lambda)$ is : 


$$
\begin{aligned}
\frac{\partial R}{\partial t}\left(t_{0}(\lambda), \lambda\right) & =R\left(t_{0}(\lambda), \lambda\right)\left(-\frac{5}{2} t_{0}(\lambda)^{-1}\right)+R\left(t_{0}(\lambda), \lambda\right)\left(-\mu_{a}(\lambda) v+\frac{\rho^{2}+z_{0}(\lambda)^{2}}{4 D(\lambda) v t_{0}(\lambda)^{2}}\right) \\
& =0
\end{aligned}
$$

Taking into account that $R\left(t_{0}(\lambda), \lambda\right)$ is a maximum and thus different from zero, it follows that:

$$
-\frac{5}{2} t_{0}(\lambda)^{-1}-\mu_{a}(\lambda) v+\frac{\rho^{2}+z_{0}(\lambda)^{2}}{4 D(\lambda) v t_{0}(\lambda)^{2}}=0
$$

Which leads to :

$$
\frac{\rho^{2}+z_{0}(\lambda)^{2}}{4 D(\lambda) v}=\frac{5}{2} t_{0}(\lambda)+\mu_{a}(\lambda) v t_{0}(\lambda)^{2}
$$

Replacing into Eq.2 yields :

$$
\begin{aligned}
\log \left(\frac{R(t, \lambda)}{R\left(t_{0}(\lambda), \lambda\right)}\right)+\frac{5}{2} \log \left(\frac{t}{t_{0}(\lambda)}\right)= & \mu_{a}(\lambda) v\left(t_{0}(\lambda)-t\right) \\
& +\left(\frac{5}{2} t_{0}(\lambda)+\mu_{a} v t_{0}(\lambda)^{2}\right)\left(\frac{1}{t_{0}(\lambda)}-\frac{1}{t}\right)
\end{aligned}
$$

This equation can be put under the following form : $\mu_{a}(\lambda)$ can be found using experimental data by solving :

$$
\mu_{a}(\lambda) G(t, \lambda)=F(t, \lambda)
$$

with :

$$
\begin{aligned}
& F(t, \lambda)=-\log (R(t, \lambda))+\log \left(R\left(t_{0}, \lambda\right)\right)-\frac{5}{2} \log \left(\frac{t}{t_{0}}\right)+\frac{5}{2}\left(1-\frac{t_{0}}{t}\right) \\
& G(t, \lambda)=v \frac{1}{t}\left(t-t_{0}(\lambda)\right)^{2}
\end{aligned}
$$

$\mu_{a}(\lambda)$ can be found by applying a multivariate method on Eq.4 where $F(t, \lambda)$ contains the values to predict and $G(t, \lambda)$ the variable $\left(\mu_{a}(\lambda)\right.$ is then the 
regression coefficient found).

Once $\mu_{a}(\lambda)$ has been evaluated, Eq. 3 enables us to obtain $\mu_{s}^{\prime}(\lambda)$ :

$$
\frac{3}{4 v}\left(\rho^{2}+z_{0}(\lambda)^{2}\right)\left(\mu_{a}(\lambda)+\mu_{s}^{\prime}(\lambda)\right)=\frac{5}{2} t_{0}(\lambda)+\mu_{a}(\lambda) v t_{0}(\lambda)^{2}
$$

which gives :

$$
\frac{3 \rho^{2}}{4 v}\left(\mu_{a}(\lambda)+\mu_{s}^{\prime}(\lambda)\right)+\frac{3}{4 v}\left(\frac{\mu_{a}(\lambda)}{\mu_{s}^{\prime}(\lambda)^{2}}+\frac{1}{\mu_{s}^{\prime}(\lambda)}\right)=\frac{5}{2} t_{0}(\lambda)+\mu_{a}(\lambda) v t_{0}(\lambda)^{2}
$$

As $\mu_{s}^{\prime}(\lambda) \gg \mu_{a}(\lambda), \frac{\mu_{a}(\lambda)}{\mu_{s}(\lambda)^{2}}$ can be neglected compared to $\frac{1}{\mu_{s}(\lambda)^{\prime}} \cdot \mu_{s}^{\prime}(\lambda)$ is then linked to $\mu_{a}(\lambda)$ by a $2^{d}$ order equation :

$$
\frac{3 \rho^{2}}{4 v} \mu_{s}^{\prime}(\lambda)^{2}+\left(-\frac{5}{2} t_{0}(\lambda)-\mu_{a}(\lambda) v t_{0}(\lambda)^{2}+\frac{3 \rho^{2}}{4 v} \mu_{a}(\lambda)\right) \mu_{s}^{\prime}(\lambda)+\frac{3}{4 v}=0
$$

\subsection{Implementation on experimental data}

An accurate determination of $t_{0}(\lambda)$ may be judged as the keystone for successful results with the suggested approach. Experimental data do however contain noise and the time-resolution is limited by the measuring apparatus. These constraints prevent the use of the measured signal maximum to find $t_{0}(\lambda)$, since measurement noise may hide the real maximum value of the time-resolved signal, likewise the apparatus time-resolution restricts $t_{0}(\lambda)$ prediction accuracy. These problems may be limited by smoothing and artificially increasing the signal time-resolution.

Let $\mathbf{R}$ be a matrix defined by $\left\{R\left(t_{i}, \lambda_{j}\right)\right\}, t_{i} \in\{1 \ldots p\}, \lambda_{j} \in\{1 \ldots q\}$, which defines a TRS measurement (fig.2). A mesh of width $w_{1}$ (odd) and length $w_{2}$ (even) is wrapped on a given part of $\mathbf{R}$. The wrapping is performed by fitting a 2 variable parabolic polynomial function of the form : $a t^{2}+b t+c \lambda^{2}+d \lambda+e t \lambda+f$ with $R(t, \lambda)$. The segment $\left(t_{i}, t_{i+1}\right)$ situated at the mesh center (bold dot line 
in figure 2) is then interpolated, where $k$ new points are added between $t_{i}$ and $t_{i+1}$ using the parabolic equation of the wrapped mesh. By applying this procedure on each part of $\mathbf{R}$, a new matrix $\mathbf{R}^{\dagger}$ of size $(p \times k) \times q$ is created. For a given wavelength $\lambda_{j}$, each $t_{i}$ is taken as candidate for $t_{0}\left(\lambda_{j}\right)$ and the correlation coefficient, $r\left(t_{i}\left(\lambda_{j}\right), \lambda_{j}\right)$, between $G\left(t, \lambda_{j}\right)$ and $F\left(t, \lambda_{j}\right)$ is calculated. Assuming Eq.4 must exist at $t_{i}=t_{0}\left(\lambda_{j}\right)$, the value for $t_{0}\left(\lambda_{j}\right)$ is chosen where the highest value of the correlation coefficient is reached, $r\left(t_{0}\left(\lambda_{j}\right), \lambda_{j}\right)$ (i.e where Eq.4 is the most verified and where $\mu_{a}(\lambda)$ has a positive value). The approach of combining Eq.4 with the above descibed implementation is named MAximum Determination for Solving Time REsolved Spectroscopy Signal (MADSTRESS).

\section{Material and methods}

\subsection{TRS Instrumentation}

Figure 3 depicts the experimental setup. The instrument has been described in detail by Abrahamsson et al. ${ }^{18}$. Briefly, a mode-locked Ti:Sapphire Laser, pumped by an Ar-ion Laser, was used to generated 100 fs pulses centered around $800 \mathrm{~nm}$ with $80 \mathrm{MHz}$ repetition rate. The laser pulses were focused into a $100 \mathrm{~cm}$ long index guiding crystal fiber (ICF)(Crystal fiber A/S, Copenhagen, Denmark). The broad band light pulses generated by non-linear effects in the ICF ranged from $750 \mathrm{~nm}$ till $1100 \mathrm{~nm}$. The light was then transferred by a set of lenses into a gradient index fiber guiding the light to the sample. Another gradient fiber, with the distal tip $\rho=6 \mathrm{~mm}$ from the irradiating one, was used to collect the light re emitted from the sample. The fibers were 
put in contact with the sample. A Streak Camera (Hamamatsu, ModelC5680) coupled to an imaging spectrometer (Chromex, Model 250IS) captured the reflected light as a function of time and wavelength $R(t, \lambda)$. The spectral resolution was $0.93 \mathrm{~nm}$ distributed over 512 pixels while the temporal resolution was 2.93 ps in the span from 0 to 1900 ps, spread over 640 pixels. Integration time was $5 \mathrm{~min}$.

\subsection{Samples}

15 Golden Delicious apples were measured using the TRS setup at an ambient temperature of $25^{\circ} \mathrm{C}$. A small part of each apple was carefully removed in order to create a flat surface for applying the fibers. The measurements were performed immediately after the preparation of the apples in order to avoid flesh drying. After the TRS measurement, the reference sugar content was measured : a drop of apple juice was taken from the scanned surface below the fibers and spread on a Euromex RD645 refractometer with $0.2^{\circ}$ Brix accuracy (i.e about $0.2 \mathrm{~g}$ of sugar per $100 \mathrm{ml}$ ).

Two other kinds of samples were also evaluated using TRS. The first one was a cup filled with $0.5 \mathrm{~mm}$ diameter fructose powder. The second was a solid tissue phantom, prepared according to Swartling et al. ${ }^{24}$. The $6.5 \mathrm{~cm}$ diameter and $5.5 \mathrm{~cm}$ high epoxy phantom contained $\mathrm{TiO}_{2}$ particles as scattering centers and toner powder as absorber.

Prior to each sample measurement, an instrumental response function was recorded by connecting the transmitting and receiving fibers to each end of a thin metal tube. This instrumental response function was used to determine time zero of the streak camera response and to measure the dispersion of the 
measured pulse due to the system characteristics.

\subsection{Linear approach implementation}

The measured signal was interpolated using the interpolation procedure described in the theory part. In order to reduce the total number of parameters to tune $\left(w_{1}, w_{2}, k\right)$, the following relations were established $w_{1}=w+1$ and $w_{2}=w$. For each pair $(w, k)$, the correlation coefficient $r\left(t_{0}\left(\lambda_{j}\right), \lambda_{j}\right)$ was evaluated for 271 wavelengths of the apple measurements. Then, the performance criterion of the chosen pair $(w, k)$ was taken as the mean of all $r\left(t_{0}\left(\lambda_{j}\right), \lambda_{j}\right)^{2}$. To improve the MADSTRESS prediction efficiency, the temporal window was chosen where the signal was significantly above zero. Using the interpolated signal and the estimated $t_{0}(\lambda), \mu_{a}(\lambda)$ was evaluated by means of a classical MLR. $\mu_{s}^{\prime}(\lambda)$ was then evaluated using Eq.5. Two solutions were obtained. The one satisfying $\mu_{s}^{\prime} \gg \mu_{a}$ was chosen.

\section{Results and discussion}

\subsection{TRS Measurements}

Figure 4-a shows the light continuum that irradiates the sample. The temporal width was about 23 ps Full-Width Half-Maximum (FWHM) and the spectral width about 300nm FWHM. The spectral profile was very sensitive to changes in the laser intensity and variations in the in-coupling efficiency into the ICF. For this reason the signal may appeared quite disrupted, but 
these fluctuations did not critically influence the evaluations as MADSTRESS uses the ratio $R(t, \lambda) / R\left(t_{0}(\lambda), \lambda\right)$. The recorded signal from one apple is shown Fig. 4-b. The temporal dispersion is very high due to the scattering inside the apple. The measured pulse length reach 1000 ps, which implies a $20 \mathrm{~cm}(!)$ light path length inside the fruit, while the input/output fibers where only distant by $6 \mathrm{~mm}$. The mean transit time of photons inside the fruit is 234 ps corresponding to a mean path of $5 \mathrm{~cm}$. Since photon path distribution in turbid media have a typical banana shape ${ }^{25}$, the mean depth probed may be estimated to $2 \mathrm{~cm}$.

\subsection{Parameters setting}

Figure 5 shows the evolution of $r\left(t_{0}, \lambda\right)^{2}$ with regard to $(w, k)$ values of the interpolated mesh. The response increased rapidly when interpolation degree, $k$, exceeds 10 pixels. A good result was found for $w=30$ pixels (mesh width). The following values were retained for the interpolation procedure : $w=30$; $k=16$.

Figure 6 shows the peak of a TRS measurement. Despite its high intensity level, the signal peak still contains non negligible noise. Maximum value of the TRS curve is hidden resulting in uncertainty for $t_{0}(\lambda)$ determination. This illustrates the $t_{0}(\lambda)$ determination problem and the interest of using $r(t, \lambda)$ as a mean for $t_{0}(\lambda)$ seeking. $r(t, \lambda)$ curve is smooth, allowing the finding of its maximum easily and without any doubt. The maximum value of $r(t, \lambda)$ seems to provide a good estimation of $R(t, \lambda)$ maximum. Without $r(t, \lambda)$ function, $t_{0}$ determination would have been harder. 


\subsection{MADSTRESS performance}

\subsubsection{Comparison of experimental and analytical data}

Figure 7-a illustrates 4 temporal dispersion curves measured on an apple. The MADSTRESS analysis has provided $\mu_{a}(\lambda)$ and $\mu_{s}^{\prime}(\lambda)$ values for each of the 4 curves. Using the diffusion equation with the estimated coefficient values, analytical TRS curves were constructed. In order to get closer to reality the width of the irradiating peak was also taken into account. The fitted curves are plotted in black in fig.7-a. The raw signal and the fitted signal are very similar and difficult to separate. However a small delay may be observed in the beginning of the rising edge of the curves. This could be due to the temporal width of the irradiating peak which is neglected in the linear approach.

In order to get a more precise idea of the accuracy of our method, the determination coefficient between the measured TRS curves, $R(t, \lambda)$, and the fitted ones, $R^{*}(t, \lambda)$, have been calculated for the 271 wavelengths of an apple measurement. The histogram of the calculated determination coefficient is presented fig.7-b. The mean values of the determination coefficient is 0.997 which is clearly a high performance. Even the lower values (0.992) demonstrates the good performance of the MADSTRESS method.

\subsubsection{Reduce scattering coefficient $\mu_{s}^{\prime}(\lambda)$}

In fig.8, the reduced scattering coefficients obtained with MADSTRESS are shown. There is large difference between $\mu_{s}^{\prime}(\lambda)$ depending on the sample. Fructose powder logically appears as the most scattering sample. One way of analyzing the results in details is to use Mie theory which states that the scattering 
coefficient may be approximated by the relation :

$$
\log \left(\mu_{s}^{\prime}(\lambda)\right)=-b \log (\lambda)+\log (a)
$$

where $a$ is proportional to the density of scattering particles and $b$ is linked to the mean size of the scattering particles ${ }^{26}$ (Mie diameter). Whereas $b$ values for the apple and the phantom were near 0.5 (resp. 0.53 and 0.58), the fructose powder $b$ value attains 1.8. Large sizes of the scattering particles of fructose may be the explanation of the slope differences. There was a difference in slope between the apple scattering coefficient and the mean of the 15 apples coefficients. We also found that $\mu_{s}^{\prime}(\lambda)$ was changing significantly from one apple to another : $18 \mathrm{~cm}^{-1}$ for the lowest $\mu_{s}^{\prime}(\lambda)$ value, $26 \mathrm{~cm}^{-1}$ for the highest scattering coefficient (not shown on the figure). These results imply that the density and size of the scattering centers vary between the apples. Yet it is well known that apple cell size and porosity are different from one apple to another. Another important comment regarding fructose $\mu_{s}^{\prime}(\lambda)$ : it does not strictly follow a linear evolution. There may be several explanations to this non-linearity. The first one is the sample morphology, which includes crystallinity that might be important. Another important property of fructose, is its light polarization ability. Hence the electric field of the scattering centers will increase the wavelength variability of the scattering coefficient by changing the scattering efficiency coefficient.

\subsubsection{Absorption coefficient $\mu_{a}(\lambda)$}

Figure 9-a presents $\mu_{a}(\lambda)$ calculated values using MADSTRESS, from which different absorption peaks may be identified.

The phantom sample has a relatively plane and linear absorption coefficient 
which was an objective during its making. However, since the exact composition of the toner powder was not known it was not possible to go further in its spectrum analysis.

The fructose presents an absorption peak at $910 \mathrm{~nm}$. This band is a C-H stretch $3^{\text {rd }}$ overtone which has already been attribute to sugar by Golic et al. ${ }^{27}$. Another wide peak, also due to fructose, is found near $1010 \mathrm{~nm}$. This peak is due to $2 \times \mathrm{C}-\mathrm{H}+3 \times \mathrm{C}-\mathrm{H}$ vibration bands. In-depth analysis also reveals a small peak at $880 \mathrm{~nm}$ corresponding to $\mathrm{C}-\mathrm{H}_{3} 3^{\text {rd }}$ overtone.

Apple $\mu_{a}(\lambda)$ contains a dominant peak at $970 \mathrm{~nm}$. This well known absorption band is due to water, composing $80 \%$ of an apples content. The width of the water peak is large due to different species of water giving rise to different absorption bands (960 $\mathrm{nm}$ and $984 \mathrm{~nm}$ ). The peak also overlaps the previous identified fructose peak, but that peak is still detectable. $880 \mathrm{~nm}$ and $905 \mathrm{~nm}$ have already been identified as fructose wavelengths 28,29 .

Another conclusion can be drawn regarding the second derivative mean spectra of the apples and fructose (fig. 9-b). The derivatives were calculated using a Stavisky Golay procedure with a window, 41 pixels wide. Fructose peaks are found at $880 \mathrm{~nm}, 910 \mathrm{~nm}$ and $1005 \mathrm{~nm}$. In all three cases the peaks are shifted in the apple spectrum. This phenomenon can be explained by water interacting with the sugar. This effect is also enhanced by changes in sample temperature ${ }^{27}$.

Using the 15 apples absorption coefficient with regard to measured sugar contents, an MLR prediction model has been calibrated using 4 wavelengths. The wavelengths ( $814 \mathrm{~nm}, 828 \mathrm{~nm}, 912 \mathrm{~nm}$ and $1005 \mathrm{~nm}$ ) were selected by a stepwise algorithm using leave-one out cross-validation. The model performance (fig.10) was very good, with a determination coefficient of 0.92 and SEC of $0.51^{\circ}$ Brix. It is interesting to note that two of the fructose wavelengths 
were selected by the stepwise procedure. The good performance of the model strengthen the observations made about the fructose peaks.

\subsection{Conclusion}

Light continuum generation using photonic crystal fibers has made it possible to efficiently conduct multi spectral time-resolved measurements. The power of the newly developed instrumentations leads to the desire to use the spectral dimension during the evaluation of the scattering and absorption coefficient of the samples, in order to improve accuracy. In this study a method for evaluation of the absorption and scattering coefficients using time-resolved reflectance measurements was investigated. The MADSTRESS method is based on a linear regression and a 2-D interpolation procedure. The method allowed us to calculate absorption coefficients and scattering coefficients of apples and fructose powder. The accuracy of the method was good enough to provide the identification of fructose absorption peaks and the construction of a calibration model predicting the sugar content of apples. MADSTRESS, as a powerful method for processing data from spectrally-continued TRS data paves the ways to accurate determination of scattering and absorption coefficients, in domains as varied as agricultural and food products, pharmaceutical, chemistry and medecine. It will serve as a reference basis for chemometricians who develop specific methods to remove the scattering signal from UV-Vis and NIR spectra. 


\section{Acknowledgments}

This work was supported by the Integrated Initiative of Infrastructure project LASERLAB-EUROPE, Contract No. RII3-CT-2003-506350.

\section{References}

1. B. Osborne and T. Fearn, Near infrared spectroscopy in food analysis (John Wiley and Sons, N.Y., 1986).

2. V. Bellon and G. Boisde, "Remote near infrared spectrometry in the food industry with the use of silica and fluoride glass fiber." in $O E^{\prime}$ Lase, p. 350 (Proc.SPIE (1055), Los Angeles, USA, 1989).

3. M. A. Dempster, B. F. MacDonald, P. J. Gemperline, and N. R. Boyer, Analytica Chimica Acta 310, 43-51 (1995).

4. V. A. McGlone, R. B. Jordan, and P. J. Martinsen, Postharvest Biology and Technology 26, 135-144 (2002).

5. V. Centner, J. VerduÁndrs, B. Walczak, D. Jouan-Rimbaud, F. Despagne, L. Pasti, D.-L. Massart, and O. de Noord, Applied Spectroscopy 54, 1620$1629(2000)$.

6. R. Barnes, M. Dhanoa, and S. Lister, Applied Spectroscopy 43, 772-777 (1989).

7. P. Geladi, D. MacDougall, and H. Martens, Applied Spectroscopy 39, 491-500 (1985).

8. B. Chance, J. Leigh, H. Miyake, D. Smith, S. Nioka, R. Greenfeld, M. Finander, K. Kaufmann, W. Levy, and M. Young, Proc. Natl. Acad. Sci. USA 85, 4971-4975 (1988). 
9. S. Jacques, Applied Optics 28, 2331-2336 (1989).

10. S. Andersson-Engels, R. Berg, A. Persson, S. Svanberg, and A. Jarlman, Opt.Lett 15, 1179-1191 (1990).

11. J. Johansson, S. Folestad, M. Josefson, A. Sparen, C. Abrahamsson, S. Andersson-Engels, and S. Svanberg, Applied Spectroscopy 56, 725-731 (2002).

12. C. Abrahamsson, J. Johansson, S. Andersson-Engels, S. Svanberg, and S. Folestad, Analytical Chemistry 77, 1055-1059 (2005).

13. J. Johansson, R. Berg, A. Pifferi, S. Svanberg, and L. Bjron, PhotoChemistry and Photobiology 62, 242-247 (1999).

14. P. Zerbini, M. Grassi, R. Cubeddu, A. Pifferi, and A. Torricelli, Postharvest Biology and Technology 25, 87-97 (2002).

15. S. Tsuchikawa and T. Hamada, Journal of Agricultural and Food Chemistry 52, 2434-2439 (2004).

16. S. Tsuchikawa, , E. Sakai, K. Inoue, and K. Miyamoto, Journal of American Society of Horticultural Science 128, 391-396 (2003).

17. M. Patterson, B. Chance, and B. Wilson, Appl. Opt. 28, 2331-2336 (1989).

18. C. Abrahamsson, T. Svensson, S. Svanberg, and S. Andersson-Engels, Optics Express 12, 4103-4112 (2004).

19. T. Farrell, M. S. Patterson, and B. Wilson, Med. Phys. 19, 879-888 (1992).

20. R. Cubeddu, A. Pifferi, P. Taroni, A. Torricelli, and G. Valentini, Med. Phys 23, 1625-1633 (1996).

21. S. J. Madsen, B. C. Wilson, M. S. Patterson, Y. D. Park, S. L. Jacques, and Y. Hefetz, Applied Optics 31, 3509-3517 (1992). 
22. L. Leonardi and D. H. Burns, Analytica Chimica Acta 348, 543-551 (1997).

23. A. Ishimaru, Wave propagation and scattering in random media (Academic press, New York, 1978).

24. J. Swartling, J. Dam, and S. Anderson-Engels, Applied Optics 42, 4612-4620 (2003).

25. S. F. F. Zeng and B. Chance, Applied Optics 34, 3826-3837 (1995).

26. J. Mourant, T. Fuselier, J. Boyer, T. Johnson, and I. Bigio, Applied Optics 36, 949-957 (1997).

27. M. Golic, K. Walsh, and P. Lawson, Applied Spectroscopy 57, 139-145 (2003).

28. K. Miyamoto and Y. Kitano, J. Near Infrared Spectrosc. 3, 227-237 (1995).

29. Y. Roggo, L. Duponchel, B. Noe, and J. Huvenne, J. Near Infrared Spectrosc. 10, 137-150 (2002). 


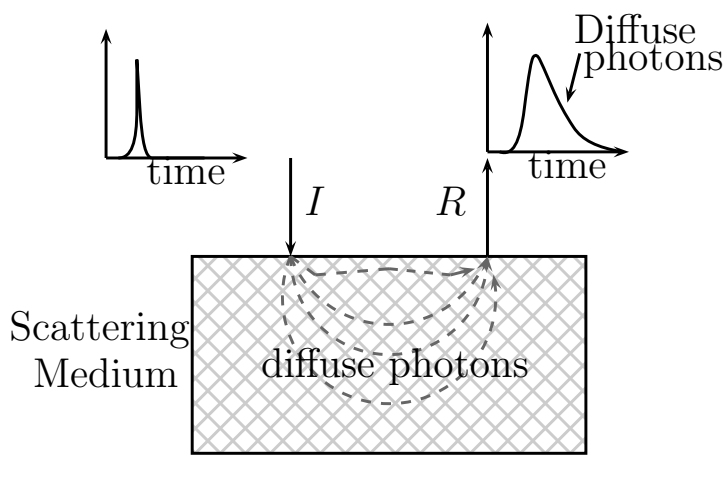

Fig. 1. Propagation of a light pulse in a turbid media 


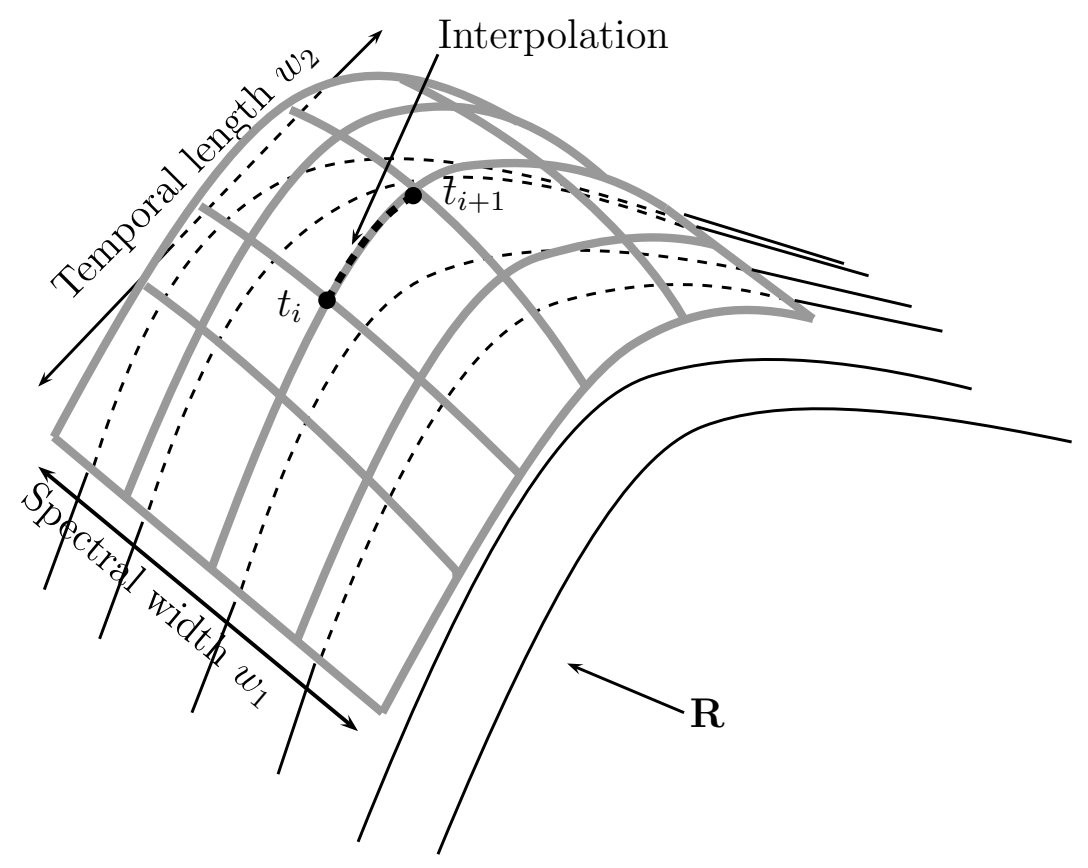

Fig. 2. Adaptative pattern for time interpolation 


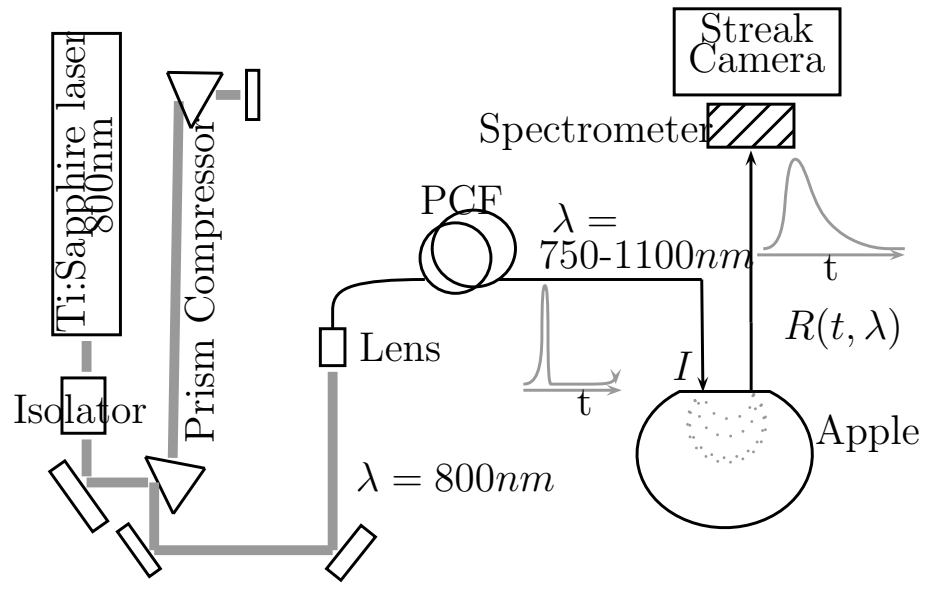

Fig. 3. Setup for TRS measurements 


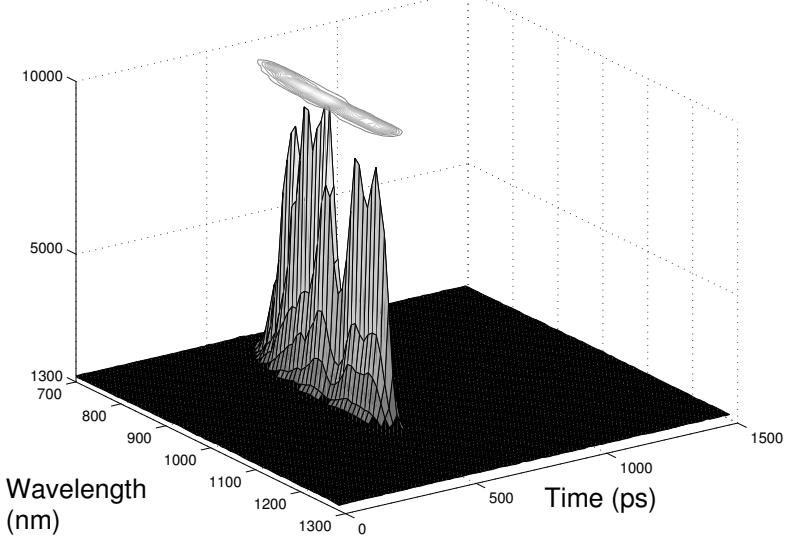

a- multispectral light pulse

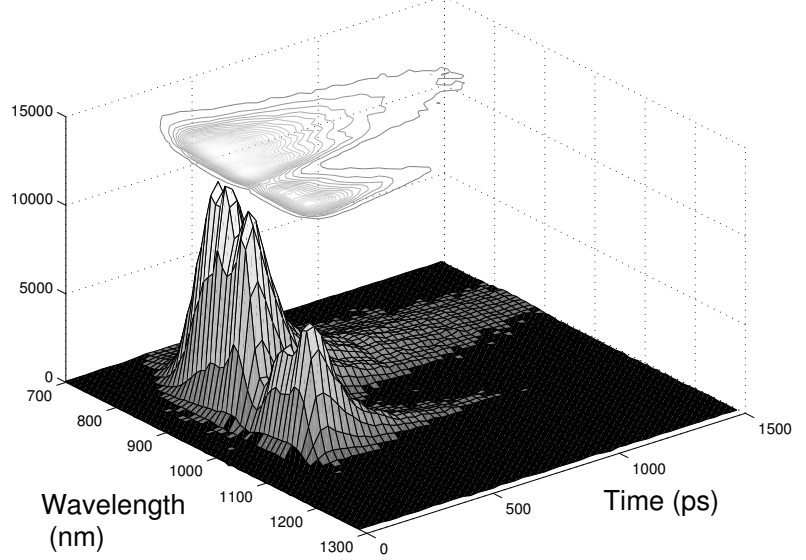

b- Signal exiting from the apple : $R(t, \lambda)$

Fig. 4. Recording of a 2D time-resolved measurement on an apple 


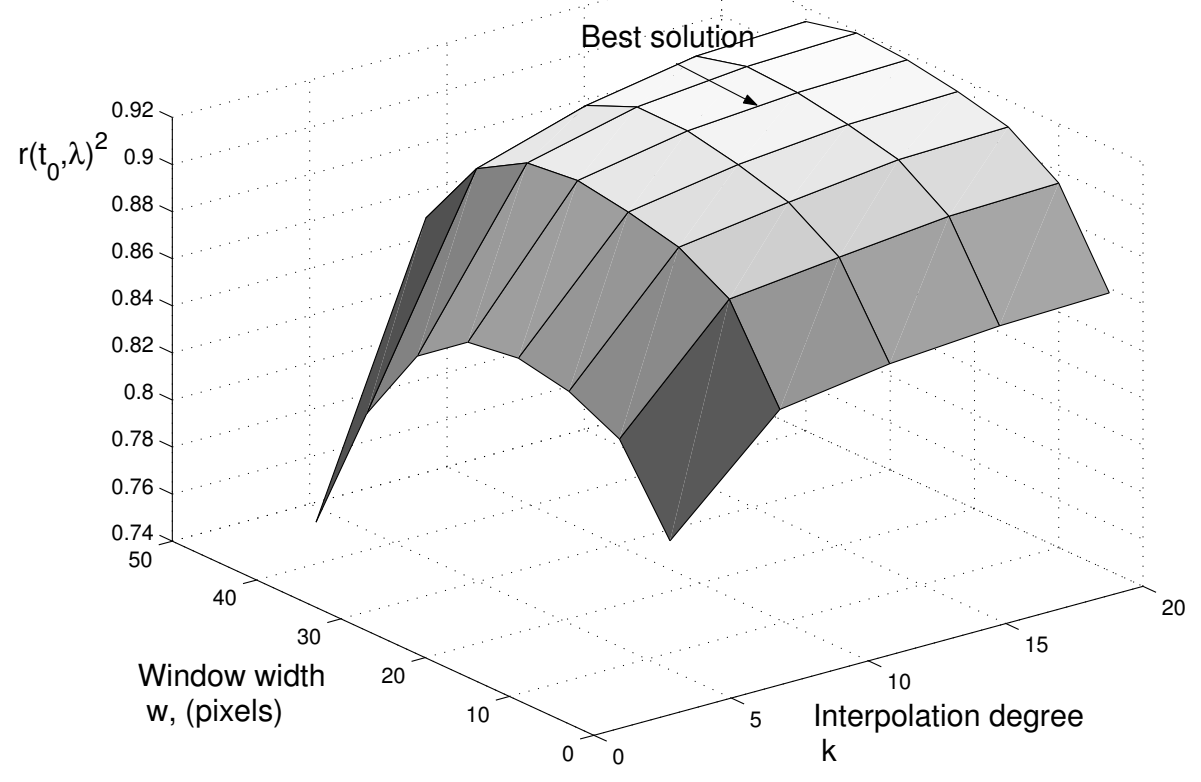

Fig. 5. $(w, k)$ tuning regarding correlation coefficient $r\left(t_{0}, \lambda\right)$ 


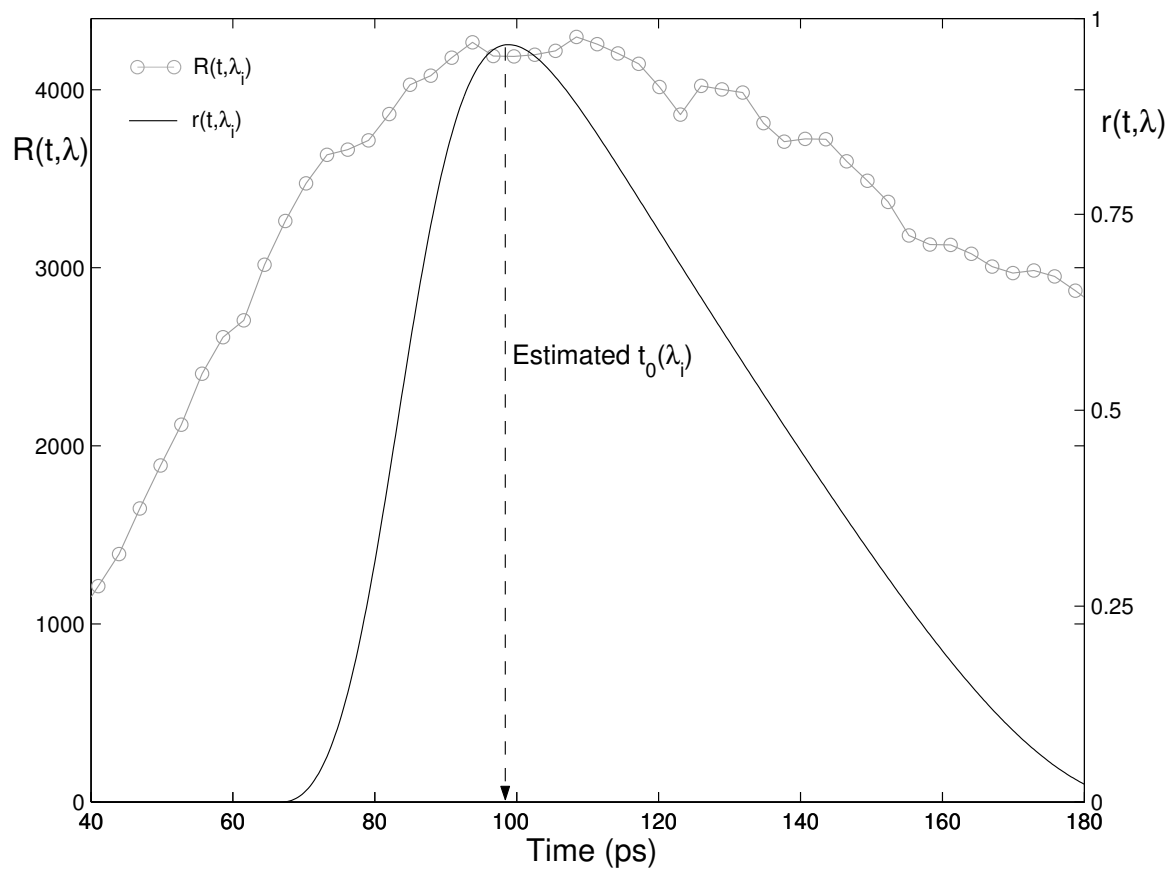

Fig. 6. Determination of $t_{0}(\lambda)$ for a TRS measurement at $\lambda=910 \mathrm{~nm}$ 


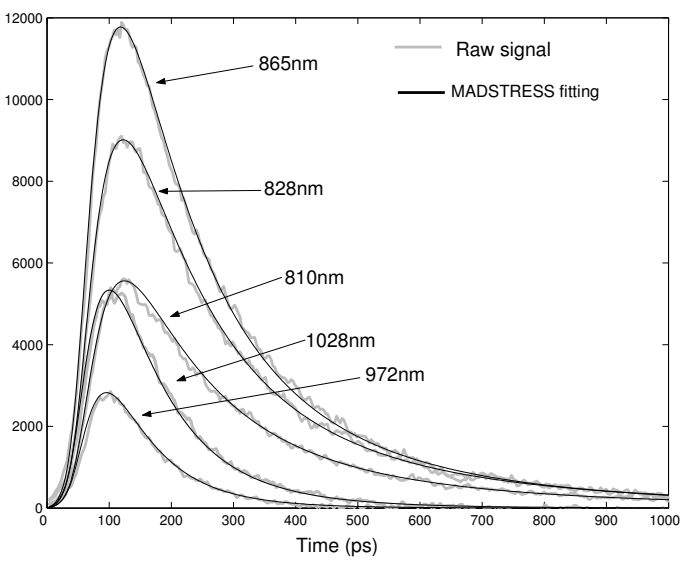

a-5 exemples

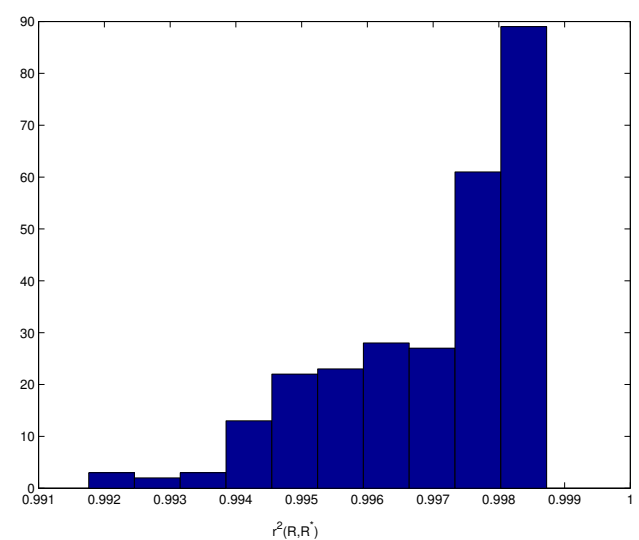

b-Histogram of determination coefficients

Fig. 7. Similarities between measured and fitted data 

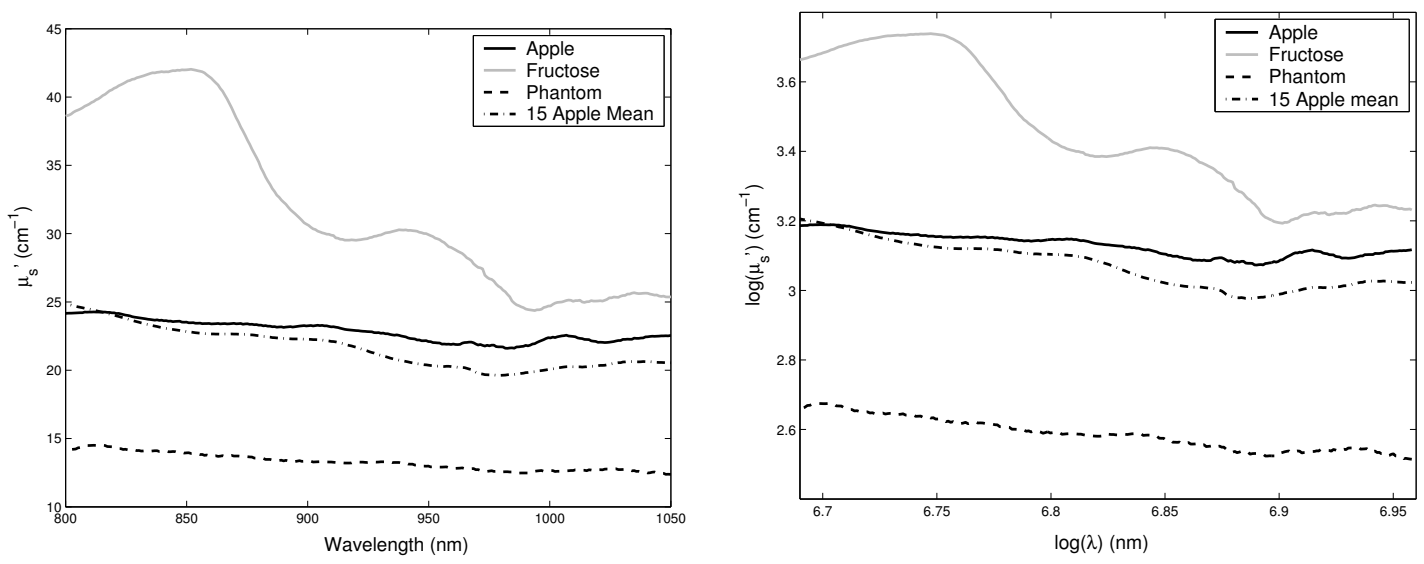

a-Normal scale

b- logarithm scale

Fig. 8. $\mu_{s}^{\prime}(\lambda)$ for the studied samples 


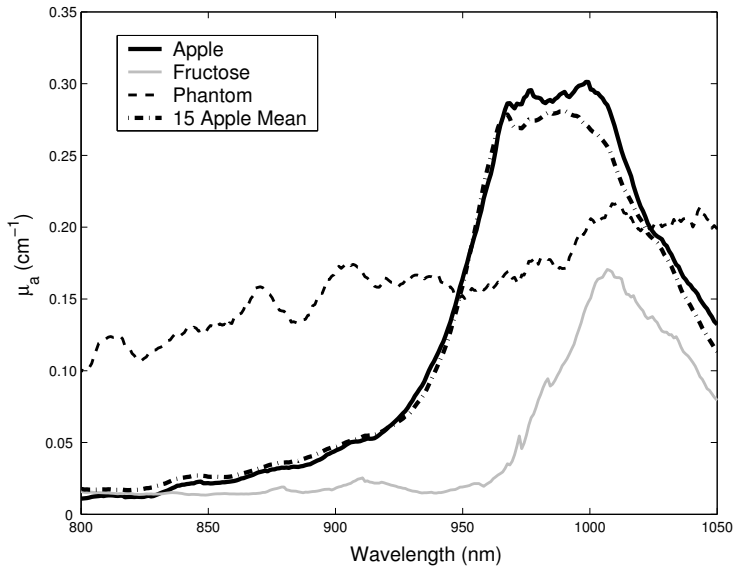

a- $\mu_{a}(\lambda)$ for the studied samples

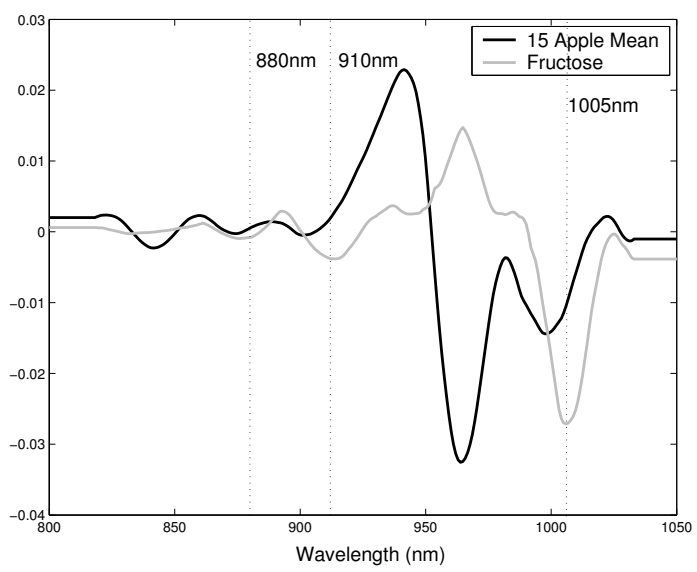

b- $2^{d}$ Derivative of apple and fructose

Fig. 9. Results of absorption coefficient evaluation 


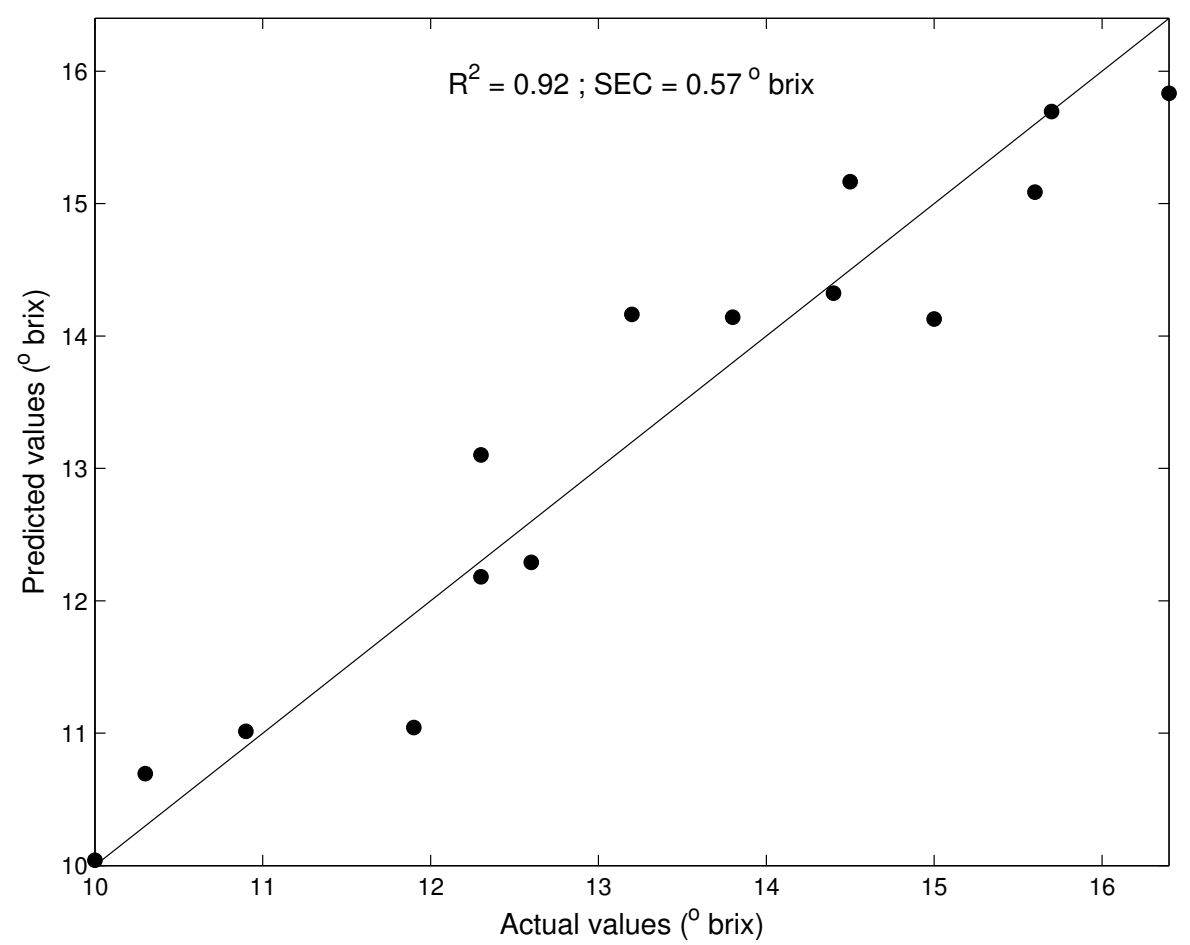

Fig. 10. Prediction of sugar concentration in apples using 4 wavelengths 